\title{
Frontières
}

\section{Limite des ressources et enjeux éthiques reliés à un traitement palliatif}

\section{Le cas de la dialyse rénale}

\section{Jocelyne Saint-Arnaud}

Volume 14, numéro 2, printemps 2002

La mort prononcée

URI : https://id.erudit.org/iderudit/1073972ar

DOI : https://doi.org/10.7202/1073972ar

Aller au sommaire du numéro

Éditeur(s)

Université du Québec à Montréal

ISSN

1180-3479 (imprimé)

1916-0976 (numérique)

Découvrir la revue

Citer cet article

Saint-Arnaud, J. (2002). Limite des ressources et enjeux éthiques reliés à un traitement palliatif : le cas de la dialyse rénale. Frontières, 14(2), 55-59.

https://doi.org/10.7202/1073972ar
Résumé de l'article

L'utilisation d'une technique palliative comme la dialyse rénale est très coûteuse en termes de budget, de personnel, de locaux et d'appareils.

Contrairement à la pratique sélective des années 1960, la pratique actuelle est ouverte à un nombre de plus en plus grand de personnes dont un pourcentage important souffre de comorbidités. Ces conditions suscitent de nombreux enjeux éthiques liés aux différentes étapes du processus de soin. Dans cet article, ces enjeux sont étudiés à partir des écrits scientifiques des dix dernières années et des résultats d'une étude exploratoire effectuées dans deux centres de dialyse québécois. 


\section{Résumé}

L'utilisation d'une technique palliative comme la dialyse rénale est très coûteuse en termes de budget, de personnel, de locaux et d'appareils. Contrairement à la pratique sélective des années 1960, la pratique actuelle est ouverte à un nombre de plus en plus grand de personnes dont un pourcentage important souffre de comorbidités. Ces conditions suscitent de nombreux enjeux éthiques liés aux différentes étapes du processus de soin. Dans cet article, ces enjeux sont étudiés à partir des écrits scientifiques des dix dernières années et des résultats d'une étude exploratoire effectuées dans deux centres de dialyse québécois.

Mots clés : dialyse - enjeux éthiques limite des ressources sanitaires

\section{Abstract}

The use of a palliative technique such as renal dialysis is cost-consuming in terms of budget, healthcare professionals, space and equipment. Contrary to the selective practice of the 1960s, the current practice is open to a greater number of people of whom a sizeable number are afflicted with co-morbidities. Such conditions give rise to many ethical issues connected to various stages in the care-giving process. In this article, these issues are studied by using both scientific writings from the past ten years and results of an exploratory study conducted at two Québec dialysis centers.

Key words : dialysis - ethical issues - limit of healthcare resources.

\title{
Limite des ressources et enjeux éthiques reliés à un traitement palliatif
}

\author{
Le cas de la dialyse rénale
}

\author{
Jocelyne Saint-Arnaud ${ }^{1}$, Ph.D. \\ professeure, Faculté des sciences infirmières, \\ Université de Montréal.
}

La préoccupation au sujet de la rareté des ressources en soins de santé est assez récente. Elle va de pair avec la crise mondiale des systèmes de santé. De nombreuses études sont entreprises pour trouver de meilleures façons de faire plus avec moins. Cependant l'utilisation de plus en plus répandue des techniques de pointe et particulièrement des techniques de maintien en vie (life-sustaining technique) est très coûteuse, quoique non remise en question, tout en suscitant des enjeux éthiques importants.

Dans ce court texte, nous allons examiner d'un point de vue historique comment se manifeste la rareté des ressources en dialyse. Dans un deuxième temps, nos présupposés méthodologiques et analytiques seront précisés. Enfin, seront analysés certains enjeux éthiques qui se présentent en cours de traitement en explicitant les liens qu'ils entretiennent avec la rareté des ressources.

\section{UN PEU D'HISTOIRE}

La technique de dialyse rénale perfectionnée en 1960 par Scribner et Quinton a suscité dès ses origines des enjeux éthiques importants. À Seattle, le nombre restreint d'appareils avait suscité la création d'un comité regroupant des médecins, des représentants d'autres disciplines et des représentants de la population. Ce comité avait pour mission de sélectionner les personnes atteintes de maladie rénale terminale qui auraient accès à la dialyse et donc qui pourraient survivre (Jonsen, 1993). Shana Alexander, une journaliste du Life Magazi$n e$ décrivant les activités de ce qu'elle avait appelé le comité de Dieu, avait intitulé son article du 9 novembre 1962: They Decide Who Lives, Who Dies; elle y avait même émis l'idée qu'il s'agissait là d'un événement fondateur de la bioéthique. En fait, la création de ce comité avait jeté les assises institutionnelles de ce qui allait devenir les comités d'éthique clinique.

Le comité de Seattle utilisait des critères médicaux et des critères sociaux pour faire le choix des neuf personnes pouvant être traitées au moyen des trois appareils disponibles. Étaient exclues les personnes atteintes de diabète, d'hypertension, de maladies de l'artère pulmonaire ou de maladies cérébrovasculaires. Étaient exclues aussi les personnes de moins de 18 ans et de plus de 45 ans; l'âge était alors considéré comme un critère médical. Les malades devaient manifester un désir de coopérer et démontrer de bonnes dispositions pour suivre les directives de soin, notamment la diète. Le comité choisissait ceux qui pourraient bénéficier le plus du traitement et apporter une plus grande contribution à la communauté. Étaient exclus les chômeurs, les criminels et les personnes habitant à l'extérieur de l'État de Washington (McCormick, 1993).

Le comité de Seattle a été grandement critiqué pour l'utilisation de critères sociaux qui reflétaient les valeurs et croyances de ses membres (Fox et Swazey, 1992; Alexander, 
1962). Consciemment ou inconsciemment, les membres de ce comité choisissaient des candidats qui leur ressemblaient (Fox et Swazey, 1992), tout en privilégiant ceux qui avaient de plus grandes responsabilités sociales. En procédant ainsi, ils tentaient de faire une sélection qui visait au plus grand bonheur pour le plus grand nombre, à l'encontre de procédures comme la loterie ou une liste d'attente selon la formule : premier arrivé, premier traité (McCormick, 1993). D'un point de vue éthique, les critères retenus pouvaient généralement être interprétés en termes utilitaristes : on favorisait les individus qui étaient les plus utiles à la société au détriment des autres. Ces procédures allaient à l'encontre du principe d'égalité d'accès aux soins et se justifiaient par la rareté des ressources.

\section{PROBLÉMATIQUE ACTUELLE}

Les choses ont changé par la suite. Le développement de la technique et l'influence des chartes des droits ont fait en sorte que le traitement est actuellement accessible à tous, particulièrement aux États-Unis et au Canada. Dans la pratique médicale de ces deux pays, même l'âge avancé ou la présence de co-morbidités ne sont plus des critères d'exclusion (Cummings, 1990 ; Auer, 1998). On note donc une forte croissance des personnes vivant sous dialyse rénale, parmi lesquelles des personnes atteintes de diabète (29\% au Québec en 1997) et de maladies cardio-vasculaires. Le nombre de patients en dialyse et ceux qui auront bénéficié d'une transplantation aura presque doublé au Québec entre 1997 et 2005 , ce qui correspond à une augmentation prévue de 5,8\% par année (Schaubel et al., 1999). Cette évaluation de la prévalence de la maladie est conservatrice. Le Conseil québécois d'évaluation des technologies prévoit plutôt une augmentation annuelle de 8,9\% (CÉTS, 1998).

Peut-on parler de rareté des ressources? Apparemment non, puisque l'accès au traitement n'est pas limité et que la cessation de dialyse se pratique peu. Cependant, les ressources en termes d'équipements, de locaux, de personnels et de financement n'augmentent pas en fonction du taux de croissance de la clientèle.

\section{PRÉSUPPOSÉS \\ MÉTHODOLOGIQUES ET ANALYTIQUES}

Une enquête est actuellement en cours dans 14 centres hospitaliers québécois. Il est trop tôt pour en donner des résultats même partiels. Ce dont nous voulons faire état ici, ce sont des enjeux éthiques reliés au traitement en explicitant les liens qu'ils entretiennent avec le phénomène de la rareté. Il s'agit ici d'un exposé des enjeux éthiques en dialyse, tels que définis dans les écrits scientifiques des dix dernières années, appuyés par les résultats d'une étude exploratoire effectuée dans deux centres de dialyse québécois. Dans cette étude, deux questionnaires ont été créés par une équipe multidisciplinaire sur la base des enjeux éthiques en dialyse rénale, tels qu'ils ont été décrits dans les écrits scientifiques et dans sept entretiens semi-structurés réalisés auprès de deux néphrologues en chef, deux infirmières chefs et une travailleuse sociale. C'est l'approche par principes (Beauchamp et al., 1994) qui est utilisée comme cadre conceptuel pour cette étude (et pour le projet dans son ensemble).

Pour les besoins de notre étude, un enjeu éthique se définit de la manière suivante: problème, question ou conflit de valeurs liés au sens de l'intervention et au respect des besoins des bénéficiaires. Le sens de l'intervention est défini par les buts thérapeutiques poursuivis. Selon Jonsen, Siegler et Winslade (1992), les buts de tout traitement sont les suivants: restauration de la santé, soulagement des symptômes incluant la détresse physique et la souffrance morale, restauration ou maintien d'une fonction compromise, sauvegarde ou prolongation de la vie, information, formation et counselling auprès des malades concernant leur condition et leur pronostic, tout en minimisant les torts. Chaque discipline y apporte ses connaissances, ses approches et ses méthodes.

Nous endossons l'idée que les disciplines de la santé ne sont pas éthiquement neutres, puisqu'elles visent toutes à une amélioration du bien-être du bénéficiaire. En ce sens, l'intervention de soin visant à rétablir ou à maintenir la santé implique déjà, à un premier niveau, l'application des principes de bienfaisance et de non-malfaisance. Le traitement ou l'intervention de soin choisi est celui ou celle qui apportera le plus de bénéfices sanitaires possibles et le moins de désavantages pour la personne directement concernée. Quand les buts ne sont plus atteints ou qu'ils sont évacués de l'acte de soin au profit de la seule technique, alors se pose la question du sens de l'intervention. Par ailleurs, répondre aux besoins du bénéficiaire par l'intervention de soin constitue l'application, à un premier niveau, du principe de justice selon la formule égalitaire classique : à chacun selon son besoin. Celui qui ne peut recevoir le soin ou le traitement approprié en temps opportun ne bénéficie pas d'une réelle égalité d'accès aux soins. Ajoutons à ces principes, le respect de l'autonomie de la personne, qui est assuré notamment dans l'information donnée et dans l'obtention du consentement libre et éclairé. Les quatre principes bioéthiques de Beauchamp et Childress (1994) structurent la description et l'analyse des enjeux éthiques en cause.

\section{LES ENJEUX ÉTHIQUES DANS LE PROCESSUS DE TRAITEMENT}

Pour présenter les enjeux éthiques, nous nous référons aux différentes étapes du processus de traitement en indiquant comment ces enjeux peuvent avoir des liens avec le phénomène de la rareté, tel qu'il a été décrit plus haut.

\section{EN DÉBUT DE TRAITEMENT}

Dans une enquête auprès de 88 infirmiers et infirmières, membres de l'American Nephrology Nurses Association (Redman et al., 1997), 69\% des conflits moraux identifiés concernaient l'arrêt ou la mise en dialyse. Trente-quatre pour cent des répondants ont signalé que les médecins entreprennent ou continuent la dialyse chez des patients qui sont atteints de manière terminale. Les écrits ne donnent guère d'indications sur des critères d'exclusion possibles. Quelques auteurs mentionnent le coma végétatif persistant (Eiser et al., 1997), la démence (Patte et al., 1994) et le très jeune âge (Buchman, 1996). Selon les résultats de notre étude exploratoire, il semble que toute personne atteinte de maladie rénale terminale et qui est apte à participer aux décisions de traitement, quelle que soit sa condition de santé, ait accès à la dialyse au Québec. Les médecins offrent le traitement et le bénéficiaire ne le refuse pas. À l'appui de cette pratique, des écrits scientifiques (Jacobs, 1996; Jaskula et al., 1994 ; Miller, 1993) indiquent qu'il est pertinent de faire un essai de dialyse, pour en évaluer les bénéfices; de plus, le patient qui a expérimenté le traitement est mieux en mesure de juger des avantages et des inconvénients qu'il en retire. Il serait donc plus éthique de proposer un essai de dialyse que de ne pas offrir le traitement. Cette pratique se justifie, même si elle va à l'encontre de la morale traditionnelle qui ne fait pas de distinction entre l'abstention et la cessation de traitement. Elle encourage cependant à l'utilisation de la technique pour elle-même sans considération des buts poursuivis et donc à une surutilisation du traitement. Pourtant, selon Kilner (1990), c'est l'utilisation de la technique qui devrait être justifiée et non son abstention. Tout ce débat amène à des considérations sur la futilité des traitements. Du point de vue de l'effet local du traitement, la dialyse est toujours utile, puisqu'elle pallie la fonction rénale pathologique, mais envisagé globalement, c'est-à-dire en fonction de la condition de santé globale du malade, le traitement peut ne pas être adéquat, notamment s'il maintient en vie une personne dont les capacités sont réduites à leur plus faible expression. De plus, la pratique de l'essai dans tous les cas et les raisons qui la justifient peuvent inciter à moins de vigilance 
dans l'application du principe du respect de l'autonomie de la personne, c'est-à-dire qu'il se pourrait que la personne soit moins bien informée, que l'on ne porte pas une attention suffisante et que l'on ne prenne pas le temps nécessaire pour mettre en place les conditions d'un consentement libre et éclairé. De toute manière, la décision de poursuivre le traitement devrait être réévaluée après un certain temps, ce qui ne semble pas se faire dans la pratique.

Déjà plusieurs problèmes d'éthique reliés au consentement libre et éclairé sont exposés dans les écrits contemporains. Dans notre étude exploratoire, la moitié des répondants (8 sur 16) affirment n'avoir reçu aucune information (2 sur 16) avant le premier traitement ou avoir reçu une information incomplète (6 sur 16). Les résultats d'une étude française (Patte et al., 1994) indiquent que certaines personnes sous dialyse reprochent aux médecins de ne pas les avoir mis au courant des conséquences indésirables engendrées par le traitement. Starzomski (1994) mentionne que les bénéfices probables sont présentés de manière optimiste, ce qui empêche une décision vraiment éclairée. Et comment parler de consentement libre quand aucun autre traitement n'est disponible sur-le-champ et qu'un refus de traitement entraîne la mort?

\section{EN COURS DE TRAITEMENT}

Le principal problème rencontré par les équipes de dialyse en cours de traitement est celui de la non-observance des directives de soins (Bernardini et al., 1997; Blake, 1997 ; Fisher et al., 1997 ; Eiser, 1996). Quarante pour cent des patients sous dialyse péritonéale ne respecteraient pas les directives de soins (Bernardini et al., 1997). Une étude pakistanaise (Safdar et al., 1995) montre que les patients «non compliants» en hémodialyse relativement à l'ingestion de liquides et à leur diète sont des hommes $(68 \%)$, plus âgés $(81 \%)$, ayant moins d'instruction $(75 \%)$, célibataires $(90 \%)$, en dépression (59\%) et ne se sentant pas responsables de leur propre bien-être $(85 \%)$. En fait, le traitement de dialyse constitue un fardeau physique et psychologique éprouvant pour le malade et pour ses proches. Selon une étude australienne (Rustomjee et al., 1996), les demandes de consultation en psychiatrie pour des patients hospitalisés dans un département de néphrologie sont de l'ordre de $17 \%$. Les raisons invoquées sont reliées au "coping» $(27 \%)$, à la dépression $(20 \%)$ et au nonrespect des directives de soins (11\%). Selon notre étude exploratoire, aucun des répondants ne réussit à respecter constamment sa diète. La majorité la respecte souvent (11/16), deux affirment la suivre rarement et un ne la suit jamais. Les principales raisons invoquées à l'appui des manquements sont la sévérité de la diète et le manque de motivation. Ces comportements peuvent avoir des impacts non négligeables sur les ressources disponibles dans des centres où aucun poste de traitement n'est disponible pour répondre à une urgence ou à une augmentation de clientèle. En effet, les personnes qui ne suivent pas leur diète peuvent nécessiter des temps plus longs de traitement, obligeant à modifier non seulement l'horaire de la personne concernée, mais aussi celui des autres patients et celui du personnel soignant. La plupart des centres ont uniformisé la durée des traitements en hémodialyse à quatre heures par traitement selon un horaire fixe. Avoir à modifier l'horaire dans de telles conditions entraîne des conséquences néfastes pour les patients et des inconvénients pour les soignants. Quand il s'agit du personnel, cela implique des coûts additionnels.

\section{EN FIN DE TRAITEMENT}

Plusieurs écrits européens interprètent le non-respect des directives de soin, particulièrement de l'horaire, comme un désir de cesser le traitement. Une étude rétrospective des dossiers de patients dialysés $(n=1639)$ entre 1964 et 1993 dans un centre australien démontre que $17 \%$ des décès étaient dus à la cessation de la dialyse (Catalano et al., 1996). Au Canada, on a évalué à $17 \%$ les décès de patients sous dialyse dus à des causes psychosociales en 1997 (Registre canadien des insuffisances et des transplantations d'organes, Rapport de 1999, p. 2-25). Les causes psychosociales regroupent les refus de traitement, les suicides et les arrêts de traitement pour d'autres raisons. On sait aussi que ceux qui cessent la dialyse ont un plus haut taux de co-morbidité et d'inconfort physique (Bajwa et al., 1996). Selon Cooper (1998), vivre sous dialyse représente un défi pour le malade et ses proches. À tout moment, ce défi peut devenir une charge trop lourde pour qu'ils puissent $\mathrm{y}$ faire face.

Des problèmes d'éthique sont liés à la cessation de la dialyse principalement à cause des conséquences mortelles qui en découlent. Peu d'études ont été faites sur les consultations effectuées auprès du malade ou de sa famille à ce propos. On sait cependant qu'un grand nombre de malades sont très ambivalents face à ce genre de décision; selon une étude américaine (Cohen et al., 1996), 61 \% des patients en dialyse préféreraient que leurs proches puissent aller à l'encontre des volontés qu'ils auraient émises antérieurement à leur inaptitude. Par ailleurs, très peu de personnes sous dialyse, particulièrement au Québec, ont rédigé des directives anticipées (Sekkarie et al., 1998). Par directives anticipées, nous entendons un testament biologique (living will), un mandat ou toute autre directive écrite permettant au patient de faire connaître ses volontés quant aux traitements de fin de vie qu'il recevra.

Les attitudes et les croyances jouent un grand rôle dans la décision de cesser un traitement de maintien en vie (Leggat et al., 1997). Ainsi, notre préenquête démontre que plus de la moitié des patients consultés ( $n=16)$ considèrent l'arrêt de dialyse comme un acte de suicide ou d'euthanasie; un seul considère qu'il s'agit d'une bonne pratique de soin et cinq ne savent pas. Sachant que la moitié d'entre eux a déjà pensé à cesser, mais qu'aucun n'en a fait la demande et que parmi ceux qui n'ont jamais pensé à cesser, six sur huit considèrent la cessation comme un acte d'euthanasie ou de suicide, il semble que les croyances morales influent sur ce genre de décision et de démarche. De plus, de nombreuses peurs, tant chez les praticiens que chez les patients, sont associées à la cessation de traitement. Parmi elles, la peur de la mort se retrouve tant chez les intervenants (Loftin et al., 1998; Rutecki et al., 1997 ; Jaskula et al., 1994) que chez les patients (Loftin et al., 1998; De Velasco et al., 1998) et leurs familles (Pfettscher, 1996). Certaines peurs sont propres à chacun des groupes. Chez les praticiens: peur de constater les limites de la thérapie (Jaskula et al., 1994), peur des poursuites (Moss, 1998 ; Jaskula et al., 1994 ; Christakis et al., 1993); chez les patients: peur de l'opposition des membres de l'équipe de soin ou des proches (Auer, 1998), peur de la douleur précédant la mort (Loftin et al., 1998); chez les familles: sentiment de culpabilité et peur de la désapprobation sociale (Pfettscher, 1996), crainte d'abandon du malade par l'équipe de soin (Jecker, 1995).

La crainte de poser un acte répréhensible, la peur de la mort et la croyance en la toute-puissance de la technique font en sorte que le traitement est poursuivi souvent jusqu'à la toute fin ou presque. Il n'est pas étonnant que les résultats d'une étude (Sekkarie et al., 1998) démontrent que 37 \% des personnes chez qui l'on cesse la dialyse sont des personnes devenues inaptes. En fait, quelle que soit la décision à prendre, une bonne communication entre le patient et le médecin apparaît comme une condition essentielle pour éliminer les fausses peurs, donner des indications claires sur les choix thérapeutiques envisageables, évaluer les avantages et les inconvénients de chacun des choix possibles. Ces démarches demandent du temps et de la disponibilité de la part du personnel soignant. Si ces conditions ne sont pas présentes parce que le personnel est insuffisant, la rareté des ressources se répercute sur la qualité des soins. 


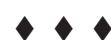

La dialyse rénale permettra de maintenir en vie un nombre de plus en plus grand de personnes dans l'avenir. Cette pratique entraîne des coûts énormes pour les systèmes de santé. On tente d'orienter davantage les patients vers la dialyse péritonéale, on organise des services de formation à l'intention des malades pour leur permettre de prendre une part plus active à leurs soins. En ce sens, on développe l'hémodialyse autonome et semi-autonome qui exige moins de personnel soignant. Cependant, l'examen des enjeux éthiques présentés ici donne des indications quant à d'autres moyens de diminuer les coûts tout en respectant les besoins du malade. Ainsi, si les personnes atteintes de maladie rénale terminale étaient informées de manière plus complète et plus objective, peut-être qu'un certain nombre d'entre elles renoncerait à la dialyse. Si certains malades ne respectent pas leur diète ou leur horaire de traitement au point d'imposer un remaniement des horaires de traitement pour les malades et les horaires de travail pour les infirmières, on pourrait les informer davantage et les sensibiliser à l'importance du respect des directives de soins. Des politiques écrites et connues à l'avance pourraient favoriser des comportements plus conformes à ces directives de soins. Enfin, il est important de dissiper les fausses peurs et la culpabilité face à la cessation de traitement. Certains malades ne savent pas qu'ils peuvent cesser un traitement et qu'une telle intervention n'est pas moralement répréhensible. Certains intervenants craignent à tort les poursuites. Une meilleure formation en éthique pour les intervenants, les patients et leur famille permettrait d'envisager la fin de manière plus sereine et d'éviter de prolonger des traitements au-delà de ce qui est humainement acceptable.

\section{Bibliographie}

ALEXANDER, S. (1962). "They decide who lives, who dies: Medical miracle puts a moral burden on a small committee », Life Magazine, vol. 53, n 9 (novembre), p. 102 et suiv.

AUER, J., (1998). "Issues surrounding the withdrawal of dialysis treatment ", Nephrology, Dialysis, Transplantation, vol. 13, p. 1149-1151.

BAJWA, K., E. SZABO et C.M. KJELLSTRAND (1996). "A prospective study of risk factors and decision making in discontinuation of dialysis ", Archives of Internal Medicine, vol. 156, $\mathrm{n}^{\circ} 22$, p. 2571-2577.

BEAUCHAMP, T.L. et J.F. CHILDRESS (1994). Principles of Biomedical Ethics, 4 éd., New York, Oxford University Press.
BERNARDINI, J. et B. PIRAINO (1997). "Measuring compliance with prescribed exchanges in CAPD and CCPD patients ", Peritoneal Dialysis International, vol. 17, $\mathrm{n}^{\circ} 4$, p. 338-342.

BLAKE, P.G. (1997). «Noncompliance in peritoneal dialysis patients», Peritoneal Dialysis International, vol. $17, \mathrm{n}^{\circ} 4, \mathrm{p} .330$ 332.

BUCHMAN, A.L. (1996). "Catheterrelated infections. », Archives of Internal Medicine, vol. 156, n ${ }^{\circ}$, p. 584.

CATALANO C, T.H. GOODSHIP, K.A. GRAHAM, C. MARINO, A.L. BROWN, J.S. TAPSON, M.K. WARD et R. WILKINSON (1996). "Withdrawal of renal replacement therapy in Newcastle upon Tyne: 1964-1993 », Nephrology, Dialysis, Transplantation, vol. 11, $\mathrm{n}^{\circ}$ 1, p. 133-139.

CHRISTAKIS N.A. et D.A. ASCH (1993). "Biases in how physicians choose to withdraw life support », Lancet, vol. 342, $n^{\circ} 8872$, p. 642-646.

COHEN, L.M., S. FISCHEL, M. GERMAIN, A. WOODS, G.L. BRADEB et J. MCCUE (1996). "Ambivalence and dialysis discontinuation", General Hospital Psychiatry, vol. $18, \mathrm{n}^{\circ} 6$, p. 431-435.

CONSEIL D'ÉVALUATION DES TECHNOLOGIES DE LA SANTÉ DU QUÉBEC (CÉTS) (1998). Hémodialyse et dialyse péritonéale: analyse comparative des rapports coûts-efficacité, Rapport CÉTS 98$3 \mathrm{RF}$, septembre.

COOPER, M.C. (1998). «Ethical decision making in nephrology nursing for end-oflife care : A responsibility and opportunity ", ANNA Journal, vol. 25, $\mathrm{n}^{\circ} 6$, p. 603-608, quiz 609-610.

CUMMINGS, N. B. (1990). "Ethical issues in geriatric nephrology: Overview », American Journal of Kidney Diseases, vol. 16, $\mathrm{n}^{\circ} 4$, p. 367-371.

DE VELASCO, R. et L.C. DINWIDDIE (1998). "Management of patient with ESRD after withdrawal from dialysis», ANNA Journal, vol. 25, n 6, p. 611-614.

EISER, A. R. (1996). "Withdrawal from dialysis: The role of autonomy and community-based values», American Journal of Kidney Diseases, vol. 27, n 3, p. 451-7.

EISER, A.R. et D.J. SEIDEN (1997). «Discontinuing dialysis in persistent vegetative state: The roles of autonomy, community, and professional moral agency ", American Journal of Kidney Diseases, vol. 30, ${ }^{\circ} 2$, p. 291-296.

FISHER, A., A. BOCK et F.P. BRUNNER (1997). "Three consecutive day collection of dialysate and urine to identify non-compliance in CAPD patients », Nephrology, Dialysis, Transplantation, vol. 12, $\mathrm{n}^{\circ} 3$, p. 570-573.

FOX, R.C. et J.P. SWAZEY (1992). "Leaving the field", Hastings Center Reports, vol. $22, n^{\circ} 5$, p. 9-15.
JACOBS, C. (1996). «Problèmes éthiques posés par les traitements de l'urémie chronique terminale», La Presse Médicale, vol. 25 , n 30, p. 1359-62.

JASKULA, S.M., E.A. FRIEDMAN et N.B. CUMMINGS (1994). "At what cost should nephrologists preserve life? », Nephrology news \& issues, vol. 8, n 11, p. 51-53, 64.

JECKER, N.S. (1995). "Medical futility and care of dying patients", The Western Journal of Medicine, vol. 163, n 3, p. 287291.

JONSEN A.R. (1993). "The birth of bioethics", Hastings Center Reports, vol. 23, n 6 (supp.), p. S1-4.

JONSEN, A.R., M. SIEGLER et W.J. WINSLADE (1992). Clinical Ethics: A Practical Approach to Ethical Decisions in Clinical Medicine, $3^{\text {e }}$ éd., New York, Macmillan.

KILNER, J.F. (1990). "Ethical issues in the initiation and termination of treatment», American Journal of Kidney Diseases, vol. $15, \mathrm{n}^{\circ} 3$, p. 218-227.

LEGGAT, J.E. Jr., W.E. BLOEMBERGEN, G. LEVINE, T.E. HULBERT-SHEARON et F.K. PORT (1997). "An analysis of risk factors for withdrawal from dialysis before death ", Journal of the American Society of Nephrology, vol. 8, n 11 , p. 1755-1763.

LOFTIN L.P. et C. BEUMER (1998). "Collaborative end-of-life decision making in end stage renal disease", ANNA Journal, vol. 25, n 6, p. 597 ; 615-617.

MCCORMICK, T.R. (1993). «Ethical issues in caring for patients with renal failure », ANNA Journal, vol. 20, $\mathrm{n}^{\circ}$ 5, p. 549-555.

MILLER, R.B. (1993). "The quandary of initiating, withdrawing dialysis» [comment], Nephrology news \& issues, vol. 7, p. $50-51$.

MOSS, A.H. (1998). «"At least we do not feel guilty": Managing conflict with families over dialysis discontinuation. ", American Journal of Kidney Diseases, vol. 31, $\mathrm{n}^{\circ} 5$, p. $868-883$.

PATTE, D., J.P. WAUTERS et F. MIGNON (1994). "Réflexions à propos de l'arrêt des traitements par dialyse", Néphrologie, vol. $15, \mathrm{n}^{\circ} 1$, p. 7-11.

PFETTSCHER, S. A. (1996). «Nephrology nurses, euthanasia, and assisted suicide», ANNA Journal, vol. 23, n 5, p. 524-5.

REDMAN, B.K., M.N. HILL et S.T. FRY (1997). «Ethical conflicts reported by certified nephrology nurses (CNNs) practicing in dialysis settings ", ANNA Journal, vol. 24, $\mathrm{n}^{\circ} 1$, p. 23-33.

Registre canadien des insuffisances et des transplantations d'organes, Rapport de 1999, vol. 1, Dialyse et transplantations rénales (1981 à 1997).

RUSTOMJEE, S. et G.C. SMITH (1996). "Consultation-liaison psychiatry to renal medicine: Work with an inpatient unit», Australian and New Zealand Journal of Psychiatry, vol. 30, n 2 , p. 229-237. 
RUTECKI, G.W., A. CUGINO, D. JARJOURA, J.K. KILNER et F.C. WHITTIER (1997). "Nephrologists' subjective attitudes towards end-of-life issues and the conduct of terminal care», Clinical Nephrology, vol. $48, n^{\circ} 3$, p. 173-180.

SAFDAR, N., H. BAAKZA, H. KUMAR et S.A. NAQVI (1995). "Non-compliance to diet and fluid restrictions in haemodialysis patients ", Journal of the Pakistan Medical Association, vol. 45, $\mathrm{n}^{\circ} 11$, p. 293-295.

SCHAUBEL, D. E., H.I. MORRISON, M. DESMEULES, D.A. PARSONS, S.S.A. FENTON (1999). «End-stage renal disease in Canada: Prevalence projections to 2005 », Canadian Medical Association Journal, vol. 160, n 11, p. 1557-1563.
SEKKARIE, M. A. et A.H. MOSS (1998). "Withholding and withdrawing dialysis: The role of physician specialty and education and patient functional status ", American Journal of Kidney Diseases, vol. 31, $\mathrm{n}^{\circ} 3$, p. 464-472.

STARZOMSKI, R. (1994). "Ethical issues in palliative care: The case of dialysis and organ transplantation », Journal of Palliative Care, vol. 10, n 3, p. 27-34.

\section{Note}

1. Cette recherche a été effectuée dans le cadre d'une subvention stratégique du Conseil de recherches en sciences humaines du Canada (1999-2002) ayant pour titre: "Enjeux éthiques reliés à la rareté des ressources en dialyse ». Chercheuse principale : J. Saint-Arnaud (Université de Montréal); cochercheurs: Louise Bouchard (Université d'Ottawa), Carmen Loiselle (Université McGill) et Pierre Verrier (Hôpital du SacréCœur de Montréal)

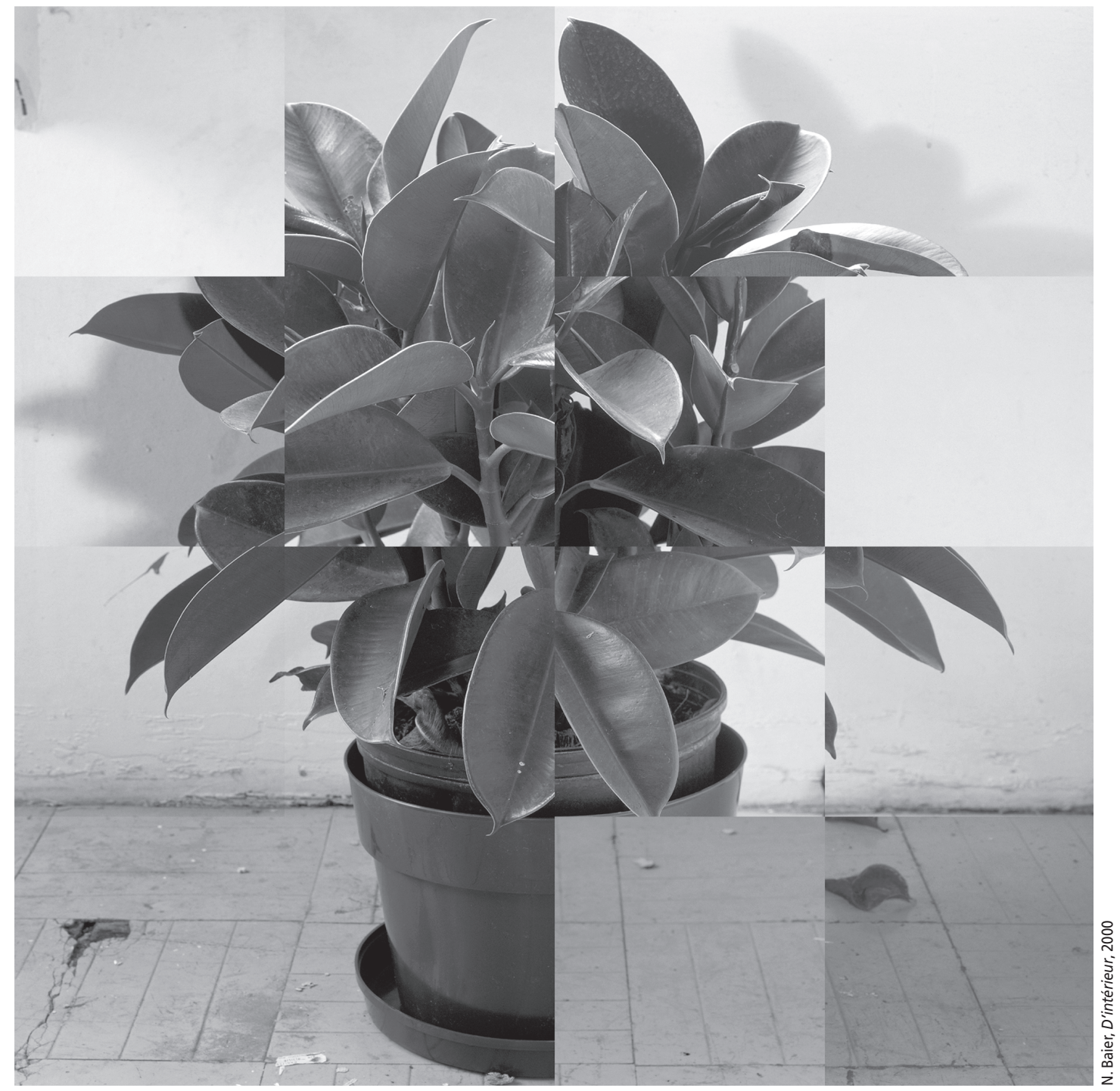

